\title{
Controlling Cherenkov Radiation with Transformation-Optical Metamaterials
}

\author{
Vincent Ginis, ${ }^{1}$ Jan Danckaert, ${ }^{1}$ Irina Veretennicoff, ${ }^{1}$ and Philippe Tassin ${ }^{2 *}$ \\ ${ }^{1}$ Applied Physics Research Group (APHY), Vrije Universiteit Brussel, Pleinlaan 2, B-1050 Brussel, Belgium \\ ${ }^{2}$ Department of Applied Physics, Chalmers University, SE-412 96 Göteborg, Sweden
}

(Received 29 December 2013; published 16 October 2014)

\begin{abstract}
In high energy physics, unknown particles are identified by determining their mass from the Cherenkov radiation cone that is emitted as they pass through the detector apparatus. However, at higher particle momentum, the angle of the Cherenkov cone saturates to a value independent of the mass of the generating particle, making it difficult to effectively distinguish between different particles. Here, we show how the geometric formalism of transformation optics can be applied to describe the Cherenkov cone in an arbitrary anisotropic medium. On the basis of these results, we propose a specific anisotropic metamaterial to control Cherenkov radiation, leading to enhanced sensitivity for particle identification at higher momentum.
\end{abstract}

DOI: 10.1103/PhysRevLett.113.167402

PACS numbers: 78.67.Pt, 41.20.Jb, 41.60.Bq

Cherenkov radiation, experimentally discovered by Pavel Cherenkov [1] and theoretically formalized by Ilya Frank and Igor Tamm [2], is a peculiar form of electromagnetic radiation that arises when charged particles travel through a medium at a velocity greater than the phase velocity of light in that medium [3]. This effect has proven useful in applied and experimental physics [4], e.g., for the detection of cosmic rays [5], novel electromagnetic sources [6-8], localized sensing in biological systems [9], spectroscopy of complex nanostructures [10], and identification of elementary particles [11]. Recently, there has been significant interest in Cherenkov radiation inside or in the vicinity of electromagnetic structured media [12-24]. This interest is fueled by the prediction that the direction of the Cherenkov cone can be reversed, such that the Cherenkov radiation and the emitting particle travel in opposite directions [25]. Aside from this experimentally observed phenomenon - called reversed Cherenkov radiation [12-17,20,21] —other unusual phenomena, such as Cherenkov radiation without a velocity threshold [24], have been predicted when charged particles travel through metamaterials and other electromagnetic structured systems [26-32].

Here, we demonstrate how the geometric techniques of transformation optics [33-37] can be used to understand the Cherenkov radiation emitted in arbitrary anisotropic media. We start by calculating the Cherenkov radiation emitted by a particle traveling along a principal axis of an anisotropic medium and we show how the resulting Cherenkov cone can be described from the threedimensional coordinate transformation in the underlying electromagnetic space. Subsequently, we discuss the physics of Cherenkov radiation in uniaxially transformed media, and we highlight the fundamental difference between transformations in directions parallel and perpendicular to the velocity of the charged particle. Finally, we demonstrate how the geometric reality of these media offers an elegant recipe for designing ring imaging Cherenkov (RICH) detectors with better resolution.

To this end, we calculate the Cherenkov radiation that is emitted by a moving charge, propagating along the $x$ axis of a medium with material parameters that correspond to a background refractive index $\left(\epsilon_{b}=n_{b}^{2}\right)$ on top of which a linear coordinate stretching along the principle axes has been implemented: $x^{\prime}=f(x), y^{\prime}=g(y), z^{\prime}=h(z)$. Following the equivalence relation of transformation optics [33,34], the material parameters of this medium are given by [38]

$$
\begin{aligned}
& \epsilon_{x, x} /\left(\epsilon_{0} \epsilon_{b}\right)=\mu_{x, x} / \mu_{0}=g^{\prime}(y) h^{\prime}(z) / f^{\prime}(x), \\
& \epsilon_{y, y} /\left(\epsilon_{0} \epsilon_{b}\right)=\mu_{y, y} / \mu_{0}=f^{\prime}(x) h^{\prime}(z) / g^{\prime}(y), \\
& \epsilon_{z, z} /\left(\epsilon_{0} \epsilon_{b}\right)=\mu_{z, z} / \mu_{0}=f^{\prime}(x) g^{\prime}(y) / h^{\prime}(z) .
\end{aligned}
$$

It can then be shown that the field component with wave vector $\mathbf{k}$ generated by a charged particle, traveling at velocity $\mathbf{v}$, will oscillate at a frequency $\omega=\mathbf{k} \cdot \mathbf{v}$ [38]. For a given frequency $\omega$, we obtain the direction of the emitted wave by matching $\omega=\mathbf{k} \cdot \mathbf{v}$ with the dispersion relation of the medium in which the charged particle is moving. The dispersion relation of the medium, defined by Eq. (1), can be found by inserting a plane monochromatic solution in the wave equation [38] and yields the following relation: $k_{x}^{2} / f^{\prime}(x)^{2}+k_{y}^{2} / g^{\prime}(y)^{2}+k_{z}^{2} / h^{\prime}(z)^{2}=\epsilon_{b} \omega^{2} / c^{2}$. We can now apply this dispersion relation to calculate the Cherenkov angle in a transformation-optical medium. Without loss of generality, we can restrict this analysis to the $x y$ plane. Writing $f^{\prime}(x)=F, g^{\prime}(y)=G, h^{\prime}(z)=H$ and defining $\alpha_{\mathrm{PH}}$ as the angle under which the electromagnetic waves are emitted [see Fig. 1(a)], we find that

$$
\tan \left(\alpha_{\mathrm{PH}}\right)=\frac{k_{y}}{k_{x}}=\frac{G}{F} \frac{\sqrt{F^{2} \epsilon_{b} \omega^{2} / c^{2}-k_{x}^{2}}}{k_{x}}=\frac{G}{F} \tan \left(\alpha^{*}\right),
$$


where $\alpha^{*}$ is the angle of Cherenkov radiation emitted in a medium with refractive index $n_{b} F$. This angle is simply given by the traditional Cherenkov formula and, hence,

$$
\alpha_{\mathrm{PH}}=\arctan \left\{\frac{G}{F} \tan \left[\arccos \left(\frac{c}{n_{b} F v}\right)\right]\right\} .
$$

This result can be better understood from a transformation-optical perspective. In physical (PH) space, the particle is moving at a velocity $v$ in the $x$ direction. Since this coordinate is stretched by a factor $F$, the particle seems to be moving at a velocity $F v$ in the underlying electromagnetic (EM) space. In this space, the particle simply travels through an isotropic medium with refractive index $n_{b}$ and, therefore, emits Cherenkov radiation with an opening cone $\theta_{\mathrm{EM}}=\arcsin \left[c /\left(n_{b} F v\right)\right]$. Translating the emitted radiation back to the physical space, the $x$ and $y$ components of the cone need to be compressed by a factor $F$ and $G$, respectively, in the electromagnetic space. The angle of the Cherenkov cone then becomes

$$
\theta_{\mathrm{PH}}=\arctan \left\{\frac{F}{G} \tan \left[\arcsin \left(\frac{c}{n_{b} F v}\right)\right]\right\} .
$$

In Figs. 1(b) and 1(c), we show the Cherenkov cones as a function of the coordinate stretching in both directions as obtained from finite-elements computer simulations [38]. The data points are in excellent agreement with Eq. (4), the analytical formula derived from the transformation optics perspective. It is also clear that a coordinate transformation in the longitudinal direction has a fundamentally different effect on the Cherenkov radiation than a transformation perpendicular to the direction of propagation. This is related to the fact that a transformation perpendicular to the trajectory of the charged particle only stretches the Cherenkov cone, whereas a transformation along the path of the particle also alters the velocity of the particle in the underlying electromagnetic space. As soon as this velocity drops below the speed of light $c$, Cherenkov radiation ceases to exist. This Cherenkov cutoff is present in Fig. 1(c), when the longitudinal scaling factor $F$ approaches $c /\left(n_{b} v\right)=0.5$. It is remarkable how the physics changes before and after a longitudinal coordinate transformation. In contrast to other transformation-optical devices, where ray trajectories are manipulated through an ingenious coordinate transformation, we show, here, that it is possible to start from a system without Cherenkov radiation and transform the nonradiating LiénardWiechert potentials into Cherenkov radiation and vice versa. This is a consequence of the longitudinal transformation scaling the particle's velocity above or below the cutoff velocity.

Obviously, an analogous relation is valid in the $x z$ plane, whose angle can be stretched by another factor

$$
\theta_{\mathrm{PH}}=\arctan \left\{\frac{F}{H} \tan \left[\arcsin \left(\frac{c}{n_{b} F v}\right)\right]\right\} .
$$

(a)
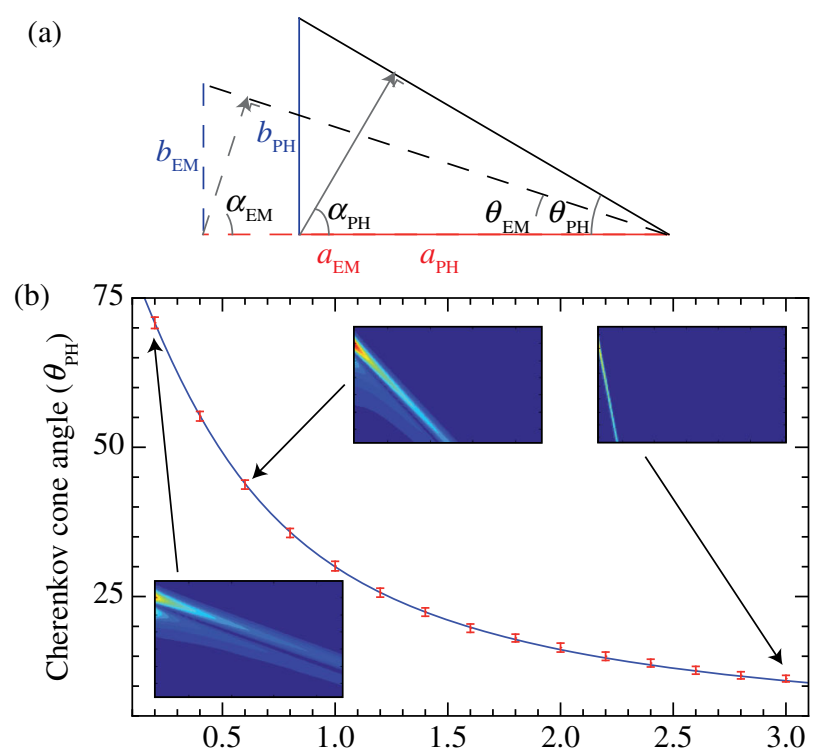

Scaling perpendicular to the propagation direction $(G)$

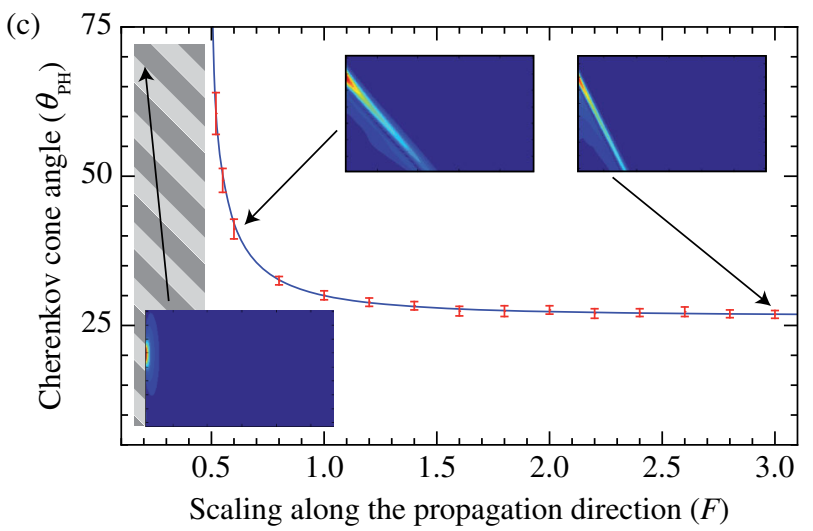

FIG. 1 (color online). (a) The definition of the angles $\alpha_{\mathrm{PH}}$ and $\theta_{\mathrm{PH}}$. The Cherenkov cone in the anisotropic physical space (solid lines) is a scaled version of the cone in electromagnetic space (dashed lines). (b)-(c) Full-wave numerical simulations of the Cherenkov radiation (red symbols) versus the corresponding analytical expression [Eq. (4)], with $F=1$ in (b) and $G=1$ in (c). In these simulations, the particle velocity and the background refractive index are related by $c / n_{b} v=0.5$. The same results are obtained for impedance-matched and for nonmagnetic implementations. The insets show the corresponding density plots of the intensity of the emitted Cherenkov radiation. The shaded area in (c) highlights the parameter regime in which no Cherenkov radiation is emitted because the velocity of the charged particle in electromagnetic space has dropped below the speed of light $\left(n_{b} F v<c\right)$.

Therefore, for a three-dimensional transformation-optical medium, Cherenkov radiation is emitted along an elliptical cone, as shown in Fig. 2. It is important to note the generality of the transformation-optical approach to studying Cherenkov radiation. First, the impedance of the material does not influence the Cherenkov cone angle. Physically, this can be understood from the fact that the cone angle is determined by constructive interference and 


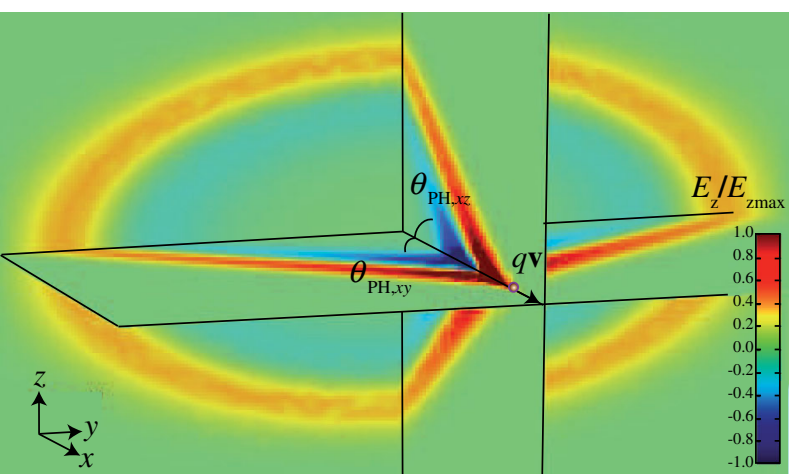

FIG. 2 (color online). The three-dimensional Cherenkov cone that is emitted in an anisotropic transformation-optical medium, obtained from full-wave simulations. The background medium implements a coordinate transformation, defined by Eq. (1) ( $n_{b}=3, F=0.7, G=0.5$, and $H=1$ ). The measured physical angles $\theta_{\mathrm{PH}, x y}$ and $\theta_{\mathrm{PH}, x z}$ are in agreement with Eqs. (4)-(5), derived from the transformation-optical perspective.

that the phase accumulation of the electromagnetic waves is solely determined by the refractive index profile and not by the impedance. We have confirmed this by comparing fullwave numerical simulations of the matched $(\epsilon=\mu)$ and the unmatched $(\mu=1)$ implementations of the coordinate transformations, and indeed, we retrieved the same angles in both sets of experiments (see Fig. 1). This is an important result from a practical point of view. Nontrivial magnetic response requires resonant electromagnetic elements, which are typically associated with large dissipative losses. The ability to implement a general coordinate transformation by solely manipulating the permittivity components, therefore, greatly reduces the dissipative losses of the material implementations. Second, inverse transformation optics allows us to predict the cone of Cherenkov radiation in any anisotropic medium by simply relating the material components to coordinate transformations.

In the second part of this Letter, we demonstrate the power of transformation optics in the design of detectors that identify unknown particles in high-energy experiments. Inside a so-called RICH detector, an unknown particle with a fixed momentum travels through a radiator and emits a Cherenkov cone. Together with the particle's momentum, the Cherenkov cone, which is a measure of the particle's velocity, allows for the determination of the mass and identification of the unknown particle. Unfortunately, the opening angle of the Cherenkov cone saturates at higher momenta to a value independent of the particle's mass: $\alpha_{\mathrm{PH} \text {,sat }}=\arccos (1 / n)$, where $n$ is the refractive index of the radiator [see Fig. 3(a)]. Consequently, it becomes very difficult to distinguish between different particles at high momentum. The traditional solution to this problem is to lower the refractive index of the radiators. This, indeed, enhances the resolving power of the detectors at higher momenta. Unfortunately, it also drastically lowers the angle under which Cherenkov radiation is emitted and,
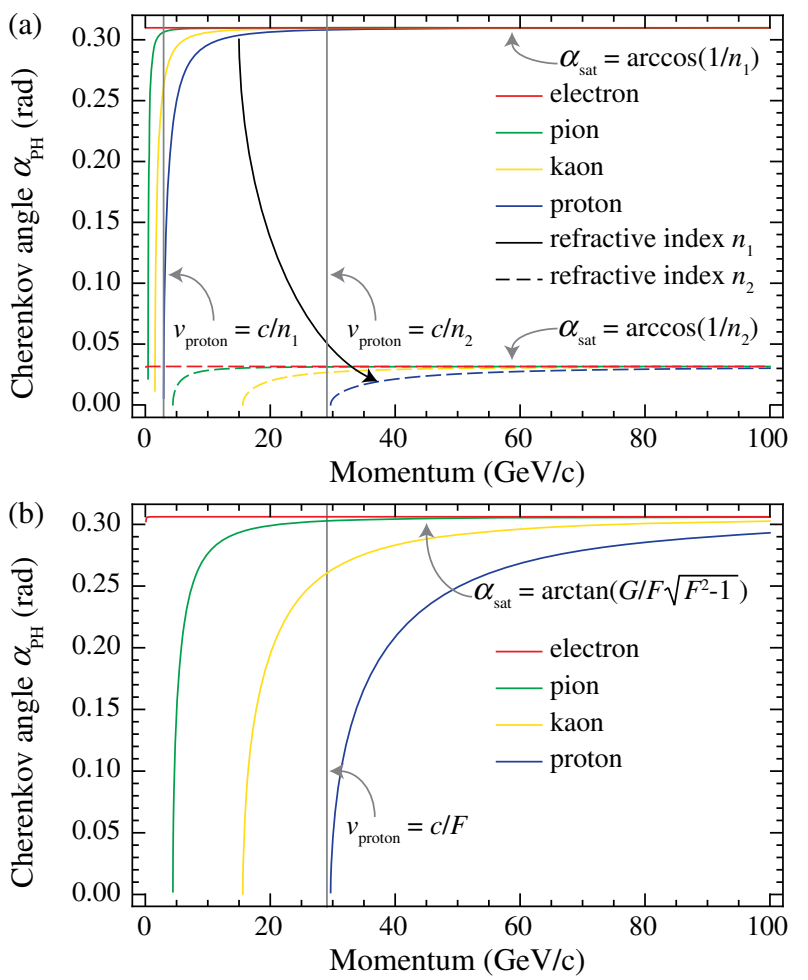

FIG. 3 (color online). (a) The Cherenkov angle versus momentum for several particles emitted inside traditional isotropic radiators: the solid lines correspond to a silica aerogel $(n=1.05)$ radiator, the dashed lines correspond to a $\mathrm{CF}_{4}(n=1.0005)$ radiator. For isotropic radiators, there exists a trade off between resolution and opening angles. (b) It is possible to combine the resolution of a $\mathrm{CF}_{4}$ radiator with the large opening angles of a silica radiator inside a transformation-optical medium in which vacuum is stretched such that $F=1.0005$ and $G=10$.

consequently, the number of photons emitted per unit length decreases [41]. This trade off between resolution and intensity is demonstrated in Fig. 3(a), where we plot the Cherenkov angle $\alpha_{\mathrm{PH}}$ as a function of the particle's momentum for several particles traveling through two traditional, isotropic radiators: one with a refractive index $n_{1}=1.05$ (silica aerogel radiator) and one with a refractive index $n_{2}=1.0005\left(\mathrm{CF}_{4}\right.$ gas radiator $)$.

Using transformation optics, we can now design a radiator that overcomes this trade off, combining enhanced resolution at higher momenta with large Cherenkov angles. As can be inferred from Fig. 3(a), the difference between the Cherenkov angles of protons and electrons-which is a good measure of the detector's resolution-is maximal close to the momentum cutoff of the proton. This cutoff corresponds to the momentum where the proton's velocity in electromagnetic space $v_{\mathrm{EM}}=v_{\mathrm{PH}} F$ equals the speed of light $c$, where we implicitly assume that the coordinate transformation acts upon a vacuum region. The best resolution can, therefore, be attained by shifting this momentum cutoff close to the momentum of the particle under investigation. Geometrically, this means that the 


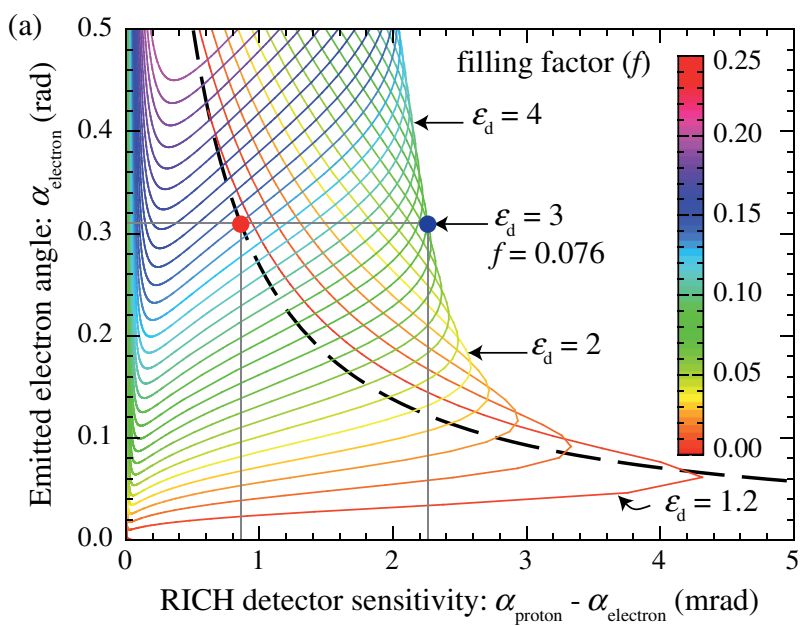

(b)

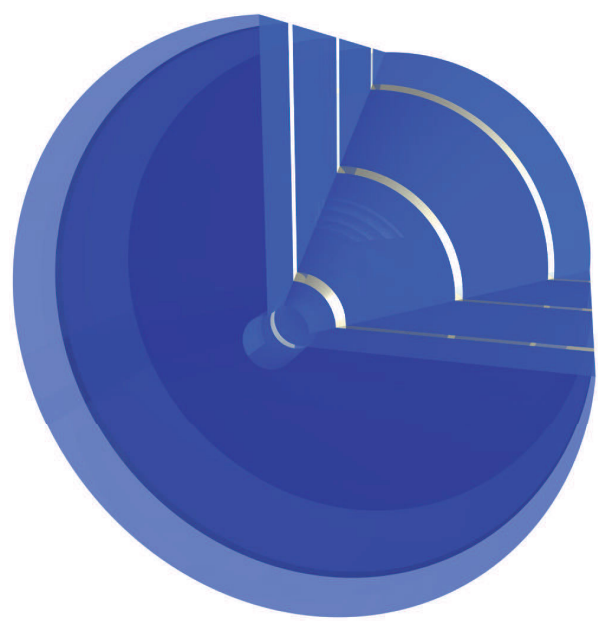

FIG. 4 (color online). (a) A comparison between traditional radiators and metamaterial radiators for fixed momentum (40 GeV/c) and wavelength $(\lambda=700 \mathrm{~nm})$. Isotropic radiators (black dashed line) suffer from a trade off between sensitivity and magnitude of Cherenkov angles. The colored lines plot the properties of several metamaterial radiators. Each line corresponds to a different background dielectric, starting from $\epsilon_{\mathrm{d}}=1.2$ at the lowest curve and increasing with steps of 0.2 for the consecutive curves. The color of the curves encodes the value of the filling factor. In order to obtain a metamaterial implementation with low dissipative losses, we have restricted the parameter regime to stay far away from the Maxwell-Garnett resonances. The colored circles show that a metamaterial with $\epsilon_{\mathrm{d}}=3$ and $f=0.076$ (blue circle) supports Cherenkov angles of the same magnitude $(\alpha=0.310$ rad) as a silica aerogel radiator (red circle), in combination with a more than twofold increased sensitivity ( $\Delta \alpha=0.86 \mathrm{mrad} \rightarrow \Delta \alpha=2.27 \mathrm{mrad}$ ). (b) An implementation of a metamaterial with parameters corresponding to the blue circle in (a): thin silver cylinders embedded in a dielectric with $f=0.076$. The central region is unfilled to allow for unobstructed propagation of charged particles. The thickness of the layers equals 20 and $234 \mathrm{~nm}$ for the silver and the dielectric, respectively. The material can be treated as an effective, homogeneous medium, so transition radiation can be neglected $[44,45]$.

longitudinal stretching $F$ should decrease as the particle's momentum increases. It is clear, from Fig. 3(b), that the curves shift to the right as $F$ becomes smaller. Although this increases the relative difference between the Cherenkov cones of the particles, it also decreases the absolute value of the cone angles. To compensate for this, we can stretch the transverse coordinates to open up the cones. This mechanism is visualized in Fig. 3(b), in which the longitudinal stretching $F$ equals 1.0005 and the transversal stretching $G$ equals 10 . We observe that this detector has the high resolution of a $\mathrm{CF}_{4}$ radiator, in combination with the Cherenkov intensity of a silica aerogel radiator.

The large anisotropy required for the implementation of these transformations is not readily available in naturally occurring materials, but can be obtained from electromagnetic structured systems [26-28,31]. An array of thin metallic cylinders embedded in a dielectric medium is a typical example of a strong anisotropic metamaterial $[42,43]$. The use of nonresonant metamaterials far away from the Maxwell-Garnett resonance reduces the spectral dispersion and dissipation present in our transformationoptical radiator. The functionality of several metamaterial radiators is evaluated in Fig. 4(a), in which we show the sensitivity and the magnitude of their Cherenkov angles as a function of the filling factor and the background dielectric at fixed momentum $(40 \mathrm{GeV} / c)$ and wavelength $(\lambda=700 \mathrm{~nm})$. The dashed line shows the trade off of isotropic radiators and runs from the top left for a high index radiator to the bottom right for a low index radiator. The other lines correspond to metamaterials in which silver cylinders are embedded in background media with various permittivities $\left(\epsilon_{\mathrm{d}}\right)$ where the filling factor is the running parameter (visualized by the color bar). The colored circles denote the enhanced resolution of metamaterial radiators with respect to the traditional ones. The red circle indicates the location of a silica radiator, whereas the blue circle corresponds to a metamaterial with a background permittivity $\epsilon_{\mathrm{d}}=3$ and a filling factor $f=0.076$, as visualized in Fig. 4(b) $\left(\epsilon_{\rho}=1.0190+0.0299 i, \epsilon_{z}=3.657+0.003 i\right)$. The overall magnitude of the opening angles is the same in both cases, but the difference between the proton and the electron angles has increased by more than twofold.

In conclusion, the work reported in this Letter demonstrates that transformation optics can be used to describe the Cherenkov cone emitted from relativistic charged particles traveling through complex anisotropic media. As an example of this technique, we calculated the material parameters that constitute particle detectors with enhanced detection sensitivity and we proposed a realistic metamaterial implementation of such a detector. We expect that these insights, together with the development of novel metamaterials, including low loss all-dielectric implementations [46-50] will allow for unprecedented control over the properties of 
Cherenkov radiation, benefiting applications in experimental and applied physics.

The authors kindly acknowledge Dr. Thomas Koschny and Dr. Freya Blekman for interesting suggestions. Work at VUB was supported by BelSPO (Grant No. IAP P7-35). V. G. acknowledges support from the Research FoundationFlanders (FWO-Vlaanderen) under Grant No. 12O9115N.

*Corresponding author.

[1] P. A. Cerenkov, Compt. Rend. Acad. Sci. USSR 8, 451 (1934).

[2] I. M. Frank and I. E. Tamm, Compt. Rend. Acad. Sci. USSR 14, 109 (1937).

[3] L. D. Landau and E.M. Lifshitz, Electrodynamics of Continuous Media (Pergamon, New York, 1960).

[4] J. V. Jelley, Cerenkov Radiation and its Applications (Pergamon, London, 1958).

[5] IceCube Collaboration, Science 342, 6161 (2013).

[6] G. Adamo, K. F. MacDonald, Y. H. Fu, C. M. Wang, D. P. Tsai, F. J. Garcia de Abajo, and N. I. Zheludev, Phys. Rev. Lett. 103, 113901 (2009).

[7] S. Liu, P. Zhang, W. Liu, S. Gong, R. Zhong, Y. Zhang, and M. Hu, Phys. Rev. Lett. 109, 153902 (2012).

[8] K. Vijayraghavan, Y. Jiang, M. Jang, A. Jiang, K. Choutagunta, A. Vizbaras, F. Demmerle, G. Boehm, M. C. Amann, and M. A. Belkin, Nat. Commun. 4, 2021 (2013).

[9] R. Robertson, M. Germanos, C. Li, G. Mitchell, S. Cherry, and M. Silva, Phys. Med. Biol. 54, N355 (2009).

[10] F. J. García de Abajo, N. Zabala, A. Rivacoba, A. G. Pattantyus-Abraham, M. O. Wolf, and P.M. Echenique, Phys. Rev. Lett. 91, 143902 (2003).

[11] J. Seguinot and T. Ypsilantis, Nucl. Instrum. Methods 142, 377 (1977).

[12] J. Lu, T. Grzegorczyk, Y. Zhang, J. Pacheco, Jr, B.-I. Wu, J. Kong, and M. Chen, Opt. Express 11, 723 (2003).

[13] C. Luo, M. Ibanescu, S. G. Johnson, and J. Joannopoulos, Science 299, 368 (2003).

[14] Y. O. Averkov and V. M. Yakovenko, Phys. Rev. B 72, 205110 (2005).

[15] S. Antipov, L. Spentzouris, W. Gai, M. Conde, F. Franchini, R. Konecny, W. Liu, J. Power, Z. Yusof, and C. Jing, J. Appl. Phys. 104, 014901 (2008).

[16] Z. Duan, B.-I. Wu, J. Lu, J. A. Kong, and M. Chen, Opt. Express 16, 18479 (2008).

[17] Z. Duan, B.-I. Wu, J. Lu, J. A. Kong, and M. Chen, J. Appl. Phys. 104, 063303 (2008).

[18] S. Xi, H. Chen, T. Jiang, L. Ran, J. Huangfu, B.-I. Wu, J. A. Kong, and M. Chen, Phys. Rev. Lett. 103, 194801 (2009).

[19] J.-K. So, J.-H. Won, M. Sattorov, S.-H. Bak, K.-H. Jang, G.-S. Park, D. Kim, and F. Garcia-Vidal, Appl. Phys. Lett. 97, 151107 (2010).

[20] M. Bakunov, R. Mikhaylovskiy, S. Bodrov, and B. Luk'yanchuk, Opt. Express 18, 1684 (2010).

[21] Z. Duan, C. Guo, and M. Chen, Opt. Express 19, 13825 (2011).
[22] S. N. Galyamin and A. V. Tyukhtin, Phys. Rev. E 84, 056608 (2011).

[23] V. V. Vorobev and A. V. Tyukhtin, Phys. Rev. Lett. 108, 184801 (2012).

[24] D. E. Fernandes, S. I. Maslovski, and M. G. Silveirinha, Phys. Rev. B 85, 155107 (2012).

[25] V. G. Veselago, Sov. Phys. Usp. 10, 509 (1968).

[26] D. R. Smith, J. B. Pendry, and M. C. K. Wiltshire, Science 305, 788 (2004).

[27] R. Engheta and R. W. Ziolkowski, Metamaterials, Physics and Engineering Explorations (IEEE, Piscataway, NJ, 2006).

[28] V. M. Shalaev, Nat. Photonics 1, 41 (2007).

[29] H. Chen, C. T. Chan, and P. Sheng, Nat. Mater. 9, 387 (2010).

[30] Y. Liu and X. Zhang, Chem. Soc. Rev. 40, 2494 (2011).

[31] C. M. Soukoulis and M. Wegener, Nat. Photonics 5, 523 (2011).

[32] N. I. Zheludev and Y. S. Kivshar, Nat. Mater. 11, 917 (2012).

[33] J. B. Pendry, D. Schurig, and D. R. Smith, Science 312, 1780 (2006).

[34] U. Leonhardt, Science 312, 1777 (2006).

[35] D. Shurig, J. B. Pendry, and D. R. Smith, Opt. Express 15, 14772 (2007).

[36] A. Greenleaf, Y. Kurylev, M. Lassas, and G. Uhlmann, SIAM Rev. 51, 3 (2009).

[37] H. Chen, R.-X. Miao, and M. Li, Opt. Express 18, 15183 (2010).

[38] See Supplemental Material at http://link.aps.org/ supplemental/10.1103/PhysRevLett.113.167402 for the details of the analytical derivations and for additional information on the computer simulations, which includes Refs. [39-40].

[39] A. Sihvola, Electromagnetic Mixing Formulas and Applications (IEEE, London, 1999).

[40] P. B. Johnson and R. W. Christy, Phys. Rev. B 6, 4370 (1972).

[41] W. R. Leo, Techniques for Nuclear and Particle Physics Experiments: A How-To Approach (Springer-Verlag, New York, 1994).

[42] A. Fang, T. Koschny, and C. M. Soukoulis, Phys. Rev. B 79, 245127 (2009).

[43] P. B. Catrysse and S. Fan, Adv. Mater. 25, 194 (2013).

[44] Z. Duan, B.-I. Wu, S. Xi, H. Chen, and M. Chen, Prog. Electromagn. Res. 90, 75 (2009).

[45] S. N. Galyamin, A. V. Tyukhtin, A. Kanareykin, and P. Schoessow, Phys. Rev. Lett. 103, 194802 (2009).

[46] J. A. Schuller, R. Zia, T. Taubner, and M. L. Brongersma, Phys. Rev. Lett. 99, 107401 (2007).

[47] G. Castaldi, S. Savoia, V. Galdi, A. Alù, and N. Engheta, Phys. Rev. B 86, 115123 (2012).

[48] G. V. Naik, V. M. Shalaev, and A. Boltasseva, Adv. Mater. 25, 3258 (2013).

[49] P. Tassin, T. Koschny, M. Kafesaki, and C. M. Soukoulis, Nat. Photonics 6, 259 (2012).

[50] P. Moitra, Y. Yang, Z. Anderson, I. I. Kravchenko, D. P. Briggs, and J. Valentine, Nat. Photonics 7, 791 (2013). 